\title{
HIV-1 Subtype Variability in Vif Derived from Molecular Clones Affects APOBEC3G-Mediated Host Restriction
}

\author{
Irene Lisovsky ${ }^{\mathrm{a}-\mathrm{c}}$ Susan M. Schader ${ }^{\mathrm{a}} \mathrm{f}$ Richard D. Sloan ${ }^{\mathrm{a}}$ Maureen Oliveira ${ }^{\mathrm{a}}$ \\ Dimitrios Coutsinos $^{a, d}$ Nicole F. Bernard ${ }^{b, c, e}$ Mark A. Wainberg ${ }^{a}$ \\ ${ }^{a}$ McGill University AIDS Centre, Lady Davis Institute, Jewish General Hospital, ${ }^{b}$ Division of Experimental Medicine, \\ McGill University, ${ }^{C}$ Research Institute of the McGill University Health Centre, ${ }^{d}$ Department of Medicine, McGill \\ University, and 'Division of Clinical Immunology and Allergy, McGill University Health Centre, Montreal, Que., Canada; \\ fDepartment of Cell and Molecular Biology, Northwestern University Feinberg School of Medicine, Chicago, Ill., USA
}

\section{Key Words}

HIV-1 subtype diversity · Vif · APOBEC3G · Viral infectivity

\begin{abstract}
Background: The host protein APOBEC $3 G$ (A3G) can limit HIV-1 replication. Its protective effect is overcome by the HIV-1 'viral infectivity factor' (Vif), which targets A3G for proteosomal degradation. Although $V$ if is considered to be essential for HIV-1 replication, the effect of Vif variability among commonly used HIV-1 molecular clones of different genetic backgrounds on viral infectiousness and pathogenesis has not been fully determined. Methods: We cloned the intact Vif coding regions of available molecular clones of different subtypes into expression vectors. $\Delta$ vif full-length HIV-1 clonal variants were generated from corresponding subtype-specific full-length molecular clones. Replicationcompetent viruses were produced in 293T cells in the presence or absence of A3G, with Vif being supplied by the fulllength HIV-1 clone or in trans. The extent of A3G-mediated restriction was then determined in a viral replication assay using a reporter cell line. Results and Conclusions: In the
\end{abstract}

absence of A3G, Vif subtype origin did not impact viral replication. In the presence of A3G the subtype origin of Vif had a differential effect on viral replication. Vif derived from a subtype $\mathrm{C}$ molecular clone was less effective at overcoming A3G-mediated inhibition than Vif derived from either subtype B or CRF02_AG molecular clones.

Copyright $\odot 2013$ S. Karger AG, Basel

Human APOBEC3G (A3G) is a member of the apolipoprotein $B$ mRNA-editing enzyme catalytic polypeptidelike family of enzymes. A3G can restrict human immunodeficiency virus type-1 (HIV-1) infection by interfering with the synthesis of minus-strand viral cDNA via cytidine deamination that results in guanine $(G)$ to adenine (A) mutations $[1,2]$. The process of G-to-A mutations can lead to viral genomic instability through introduction of amino acid substitutions and premature stop codons. HIV-1 has evolved to overcome this restriction by expressing and packaging a virus-encoded protein termed viral infectivity factor (Vif), which induces the polyubiquitylation and subsequent proteasomal degradation of A3G $[3,4]$.

\section{KARGER}

E-Mail karger@karger.com

www.karger.com/int
(C) 2013 S. Karger AG, Basel

0300-5526/13/0564-0258\$38.00/0
Mark A. Wainberg

McGill AIDS Centre, Jewish General Hospital

3755 Chemin Côte-Sainte-Catherine

Montreal, QC H3T 1E2 (Canada)

E-Mail mark.wainberg@mcgill.ca 
Given the rapid HIV-1 replication rate and high virus titers, genetically distinct allelic variants of vif can often be detected within infected individuals $[5,6]$. Proviral DNA from some viruses that failed to neutralize A3G was shown to contain an abundance of G-to-A hypermutations [7], suggesting that sporadic inactivation of Vif can occur in vivo and that natural Vif variability might affect the course of HIV disease. HIV-1 subtype variability is extensive and ranges between 15-20 and $25-35 \%$ at the amino acid and nucleotide levels, respectively $[8,9]$. The contribution of Vif amino acid variability among HIV-1 subtypes to viral pathogenesis is not fully understood. Indeed, others have shown that the subtype origin of Vif may play a role in determining the extent to which A3G-mediated restriction of HIV replication will occur $[10,11]$. However, the impact of Vif variability among a number of commonly used fulllength subtype-specific HIV-1 infectious molecular clones has not been investigated in the context of A3Gmediated restriction.

We performed a phylogenetic analysis to determine the extent of inter-subtype genetic differences present in Vif protein and to ensure that each molecular clone used in this study was representative of other Vif sequences of the same subtype. HIV-1 vif nucleotide sequences ( $\mathrm{n}=$ 1,004), obtained through the Los Alamos HIV Database, were aligned and translated using VectorNTI software (Version 10.3.1; Invitrogen). Subtype-specific protein reference sequences were derived using Contig maker VectorNTI for subtypes A, B, C, G and A/G. Translated subtype-specific Vif sequences were realigned on VectorNTI and loaded into MEGA 4.0 software for phylogenetic analysis. The analysis revealed $80-85 \%$ similarity between subtypes B, C and CFR02_AG Vifs at the amino acid level (fig. 1). The Vif from molecular clone pAG_97 showed greater similarity to subtype A than G, which is in agreement with a recent report [12]. The Vif sequences investigated in this study are shown in figure 2; each was confirmed to be representative of its subtype of origin (fig. 1).

To assess the impact of $A 3 G$ restriction on the replication of viruses derived from subtype-specific molecular clones, the TZM-bl luciferase reporter assay system was used as previously described [13]. Recombinant viruses were produced by transfecting 293T cells with $9 \mu \mathrm{g}$ pAG_97 or pINDIE_C $\Delta v i f, 3 \mu \mathrm{g}$ Vif and $\pm 3 \mu \mathrm{g}$ pcDNA3.1-APOBEC3G using Lipofectamine 2000 (Invitrogen). Empty pcDNA3.1/V5-HisA was used as a filler control. A total of $16 \mu \mathrm{g}$ of DNA was transfected, except in the Vif titration experiment where $5 \mu \mathrm{g}$ of Vif was transfected. Supernatants were harvested $48 \mathrm{~h}$ posttransfection, clarified by centrifugation, passed through a $0.45-\mu \mathrm{m}$ filter and tested for reverse transcriptase (RT) activity. A subtype $\mathrm{C}$ viral backbone was employed to establish the assay based on current understanding of subtype B viruses and A3G restriction. To obtain \% HIV-1 replication, we normalized the measured luciferase activity for each virus tested to the assay maximum output point set by the virus derived from pNL43 produced in the presence of A3G. The results of figure 3a clearly distinguish between replication of full-length and $\Delta v$ if subtype $\mathrm{C}$ viruses produced in the presence or absence of A3G. While in the absence of A3G both the full-length subtype $\mathrm{C}$ and $\Delta$ vif subtype $\mathrm{C}$ viruses supported HIV replication, a marked reduction in HIV-1 replication was observed for $\Delta v$ vif subtype $\mathrm{C}$ virus produced in the presence of A3G (fig. 3a). This suggests that Vif encoded by the full-length subtype $\mathrm{C}$ clone was able to overcome A3G restriction which restored viral replication to levels significantly higher than seen with $\Delta v i f$ subtype C (fig. 3a, dashed lines, $\mathrm{p} \leq 0.0001$ ).

We next assessed the replication of $\Delta v$ if subtype $\mathrm{C}$ viruses complemented with Vif in trans from different viral subtypes. In the absence of $A 3 G$, the subtype source of Vif did not impact levels of viral replication (fig. 3b). Next we titrated the amount of Vif supplied in trans for virus production. With a subtype $C$ virus, increasing the amount of Vif derived from either subtype B or C from 1 to $5 \mu \mathrm{g}$ resulted in an increase in levels of viral replication (fig. $3 \mathrm{c}$ and d, respectively). Supplementation of $\Delta v$ if subtype $\mathrm{C}$ with $5 \mu \mathrm{g}$ of Vif in trans from either subtype $\mathrm{B}$ or $\mathrm{C}$ resulted in comparable replication of the Vifdeficient viruses (fig. 3c, d). Accordingly, an optimal concentration of Vif was set at $5 \mu \mathrm{g}$ and maintained in subsequent analyses.

Vif from 3 distinct subtypes complemented $\Delta v$ if subtype $\mathrm{C}$ clonal virus replication in the absence of $\mathrm{A} 3 \mathrm{G}$ at levels between 70 and $90 \%$ of maximum values (fig. $4 \mathrm{a}$, solid lines). In the presence of $\mathrm{A} 3 \mathrm{G}$, a notable reduction in HIV-1 replication was observed at levels ranging from 20 to $30 \%$ of maximum (fig. $4 \mathrm{a}$, dashed lines). The presence of Vif from the subtype B and CRF02_AG clones afforded a greater advantage to virus replication in the presence of $\mathrm{A} 3 \mathrm{G}$ compared to Vif from subtype $\mathrm{C}$ clone (fig. 4a). A similar trend was observed using recombinant viruses derived from the CRF02_AG backbone (fig. 4b). In the absence of $A 3 G$, Vif from subtype $C$ and CRF02_AG complemented $\Delta v$ if $\mathrm{AG}$ virus replication to 60 and $40 \%$ of maximum, respectively (fig. $4 \mathrm{~b}$, solid lines). However, the Vif-complemented $\Delta$ vif AG viruses, 


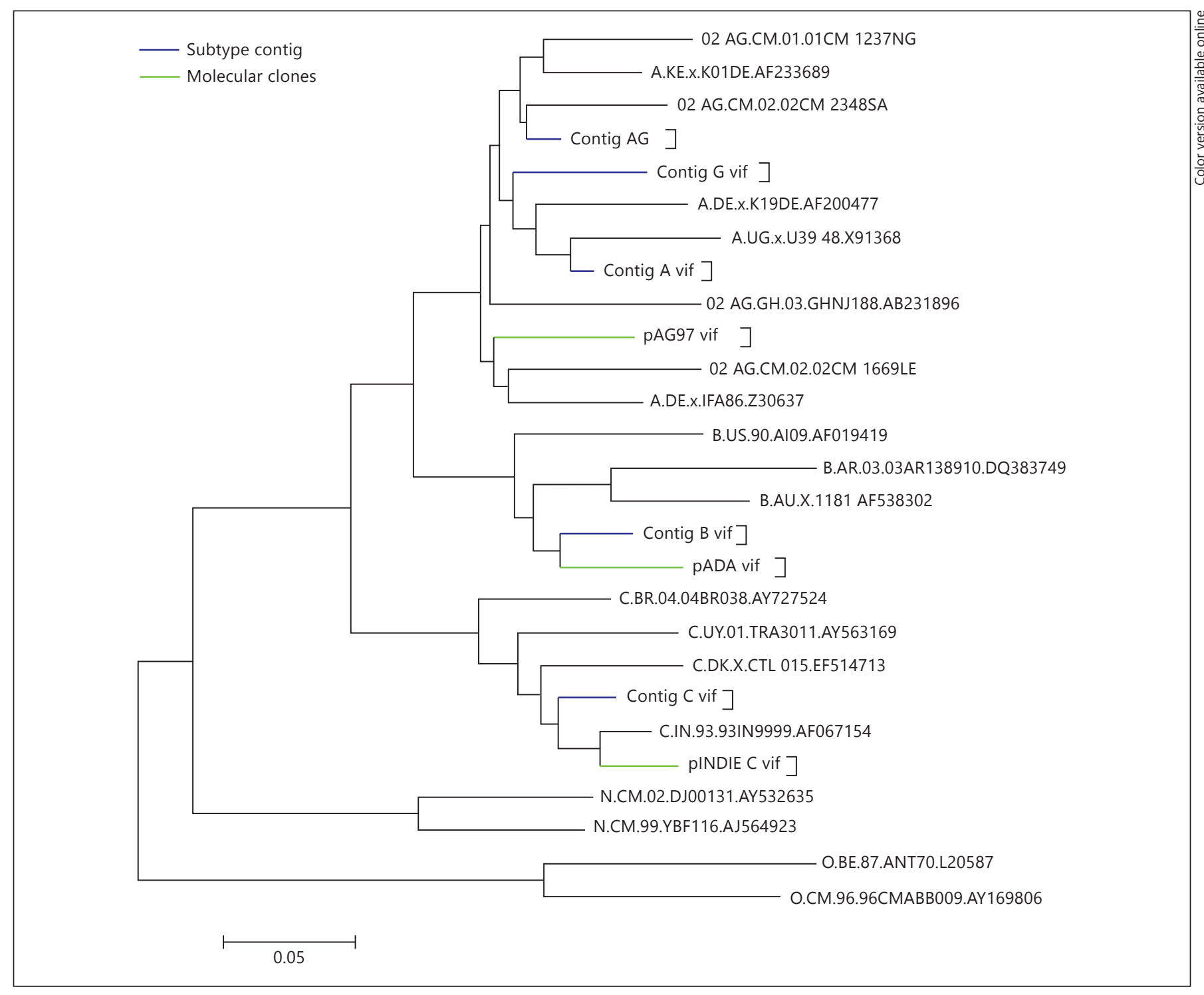

Fig. 1. Phylogenetic analysis of Vif amino acid sequences. Phylogenetic analysis of available HIV-1 vif nucleotide sequences $(\mathrm{n}=1,004)$ and subtype-specific molecular clones used in this study. The vif nucleotide sequences were aligned and translated using VectorNTI software. The resultant amino acid sequences were realigned in VectorNTI and the phylogenetic analysis was performed using the

produced in the presence of A3G, showed reduced levels of replication, ranging from 5 to $20 \%$ of maximum (fig. 4b, dashed lines). Furthermore, Vif from the subtype B and CRF02_AG clones overcame A3G-mediated restriction more effectively than Vif from subtype $\mathrm{C}$ as shown by a greater replicative advantage.

In summary, we have shown that recombinant viruses generated from different genetic backbones, in the presence of $A 3 G$, exhibited different abilities to replicate
MEGA 4 program. Subtype Contig (consensus) sequences were derived from all available vif nucleotide sequences belonging to their respective subtype or circulating recombinant forms (CRF). Homology among subtypes B, C and CRF02_AG ranged between 80 and $85 \%$ at the amino acid level. Vif amino acid sequences from HIV-1 groups $\mathrm{N}$ and $\mathrm{O}$ were also included in this phylogenetic analysis. in TZM-bl cells. We have also shown that Vif-containing viruses derived from pNL/ADA or pAG_97 were able to overcome $A 3 G$ restriction more efficiently than Vif derived from pINDIE_C (fig. 4a, b). Our study supports and extends earlier works that used chimeric proteins, that reported that Vif proteins derived from HIV-1 clinical and viral isolates of different subtypes varied in their activities against $A 3 G[10,11]$. In contrast to our results, others noted that Vif derived from subtype $\mathrm{C}$ had higher 


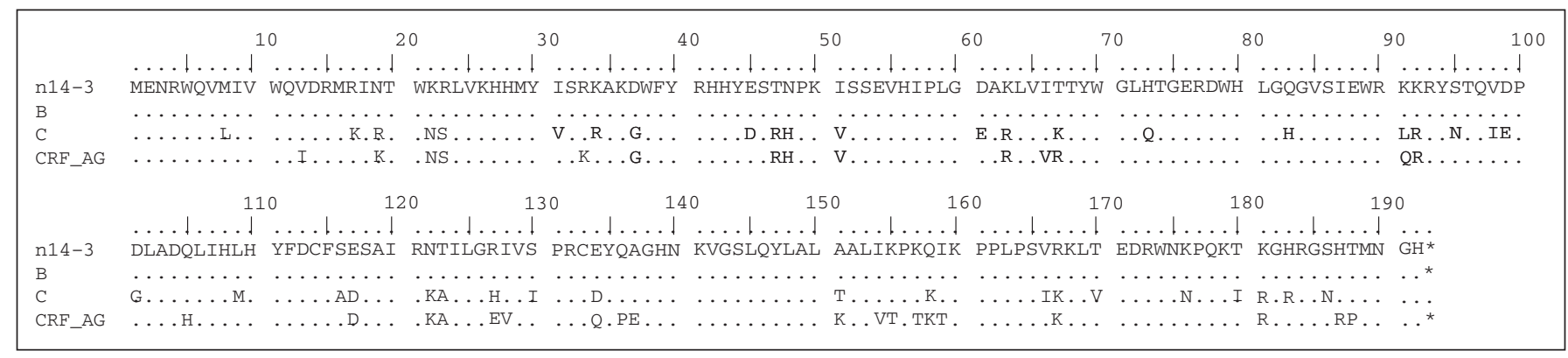

Fig. 2. HIV-1 Vif amino acid sequence alignment of the Vif clones used in this study. pNL4-3 (nl4-3) was used as a reference sequence for alignment. The Vif amino acid sequences of these molecular clones pNL/ADA (B), pINDIE_C (C) and pAG_97
(CRF_AG) were derived by translating in house sequencing data of vifs that were cloned into the mammalian expression vectors. Amino acid sequences were aligned using ClustalW alignment in Bioedit.

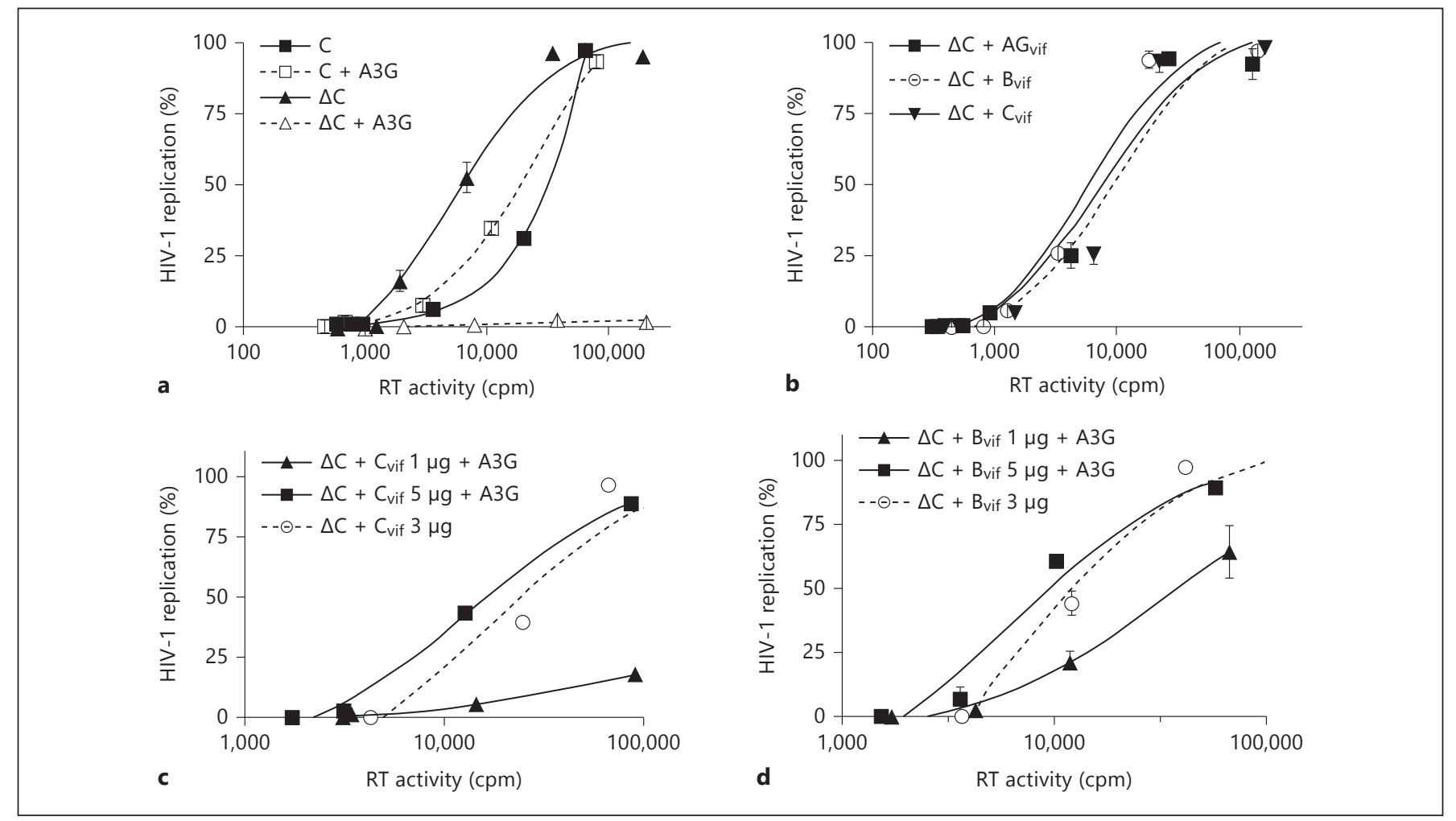

Fig. 3. Replication of HIV-1 in the presence and absence of A3G. a A3G-TZM-bl HIV-1 replication assay. Vif-deficient ( $\Delta$ vif) clones were generated by introducing nonsense mutations using a site-directed mutagenesis kit (Stratagene) at codons 26 and 27 of vif. The resultant clones were sequenced to confirm the presence of the stop codons. Replication of subtype $\mathrm{C}$ viruses derived from the pINDIE_C full-length $(\mathrm{C})$ or vif-deleted $(\Delta \mathrm{C})$ molecular clones were evaluated in the absence and presence of A3G. Luciferase readout was used to determine the extent of HIV-1 replication. b Replication of vif-deleted recombinant HIV-1 in the TZM-bl assay. Vif coding sequences from subtype-specific molecular clones were cloned into BamHI and EcoRI (NEB) digested pcDNA3.1/ V5-HisA (Invitrogen). A 5' His-tag was introduced into the clones using SDM. Clones were confirmed by digestion, sequencing and Western blotting. Vif derived from each of subtype B $\left(\mathrm{B}_{\mathrm{vif}}\right), \mathrm{C}$ $\left(\mathrm{C}_{\text {vif }}\right)$, or CRF02_AG $\left(\mathrm{AG}_{\mathrm{vif}}\right)$ molecular clones was supplied in trans to complement the $\Delta v$ if $(\Delta \mathrm{C})$ molecular clone during virus production. All viruses produced were in A3G-free conditions. c, $\mathbf{d}$ Dose response of vif-deleted pINDIE_C $(\Delta \mathrm{C})$ to A3G. Recombinant virus was generated using $\Delta \mathrm{C}$ backbone with $\mathrm{Vif}$ supplied in trans on an expression plasmid from subtype $\mathrm{C}\left(\mathrm{C}_{\mathrm{vif}}\right)$, shown in c, or B $\left(\mathrm{B}_{\mathrm{vif}}\right)$, shown in $\mathbf{d}$. To obtain \% HIV-1 replication we normalized the measured luciferase activity for each virus tested to the assay maximum output point set by virus, pNL4-3 (produced in the presence of $A 3 G$ ). All data depict the results of 3 independent experiments performed in duplicate \pm SD. 


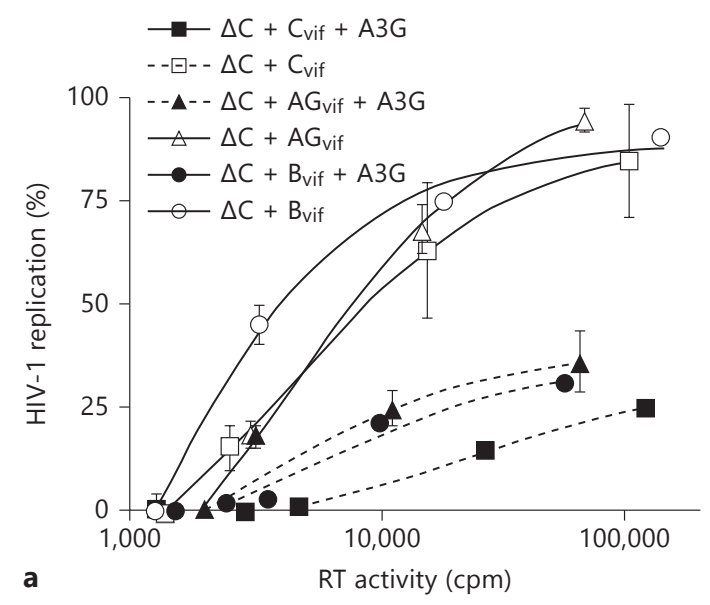

Fig. 4. Impact of Vif variability on A3G-mediated antiviral activity and HIV-1 replication. Effect of Vif subtype variability on A3G-mediated antiviral activity using the $\Delta \mathrm{C}(\mathbf{a})$, or the $\Delta \mathrm{AG}$ viral backbone (b) where Vif was supplemented in trans from subtype B $\left(\mathrm{B}_{\mathrm{vif}}\right), \mathrm{C}\left(\mathrm{C}_{\mathrm{vif}}\right)$ or CRF02_AG $\left(\mathrm{AG}_{\mathrm{vif}}\right)$. The solid lines

anti-A3G activity than those from subtypes A, B, D and CRF01_AE and CRF02_AG viruses that were tested [11]. In our study, Vif derived from the molecular subtype $\mathrm{C}$ clone was the least efficient among the Vifs tested in overcoming A3G restriction (fig. 4a, b). Previous work showed that Vif proteins derived from three distinct subtype $\mathrm{C}$ viral isolates varied in anti-A3G activity. Variations in the anti-A3G activity of Vif may be due to amino acid sequence differences between the subtype C Vif molecules used in these studies. In fact, Vif derived from the pINDIE_C clone, used here, shares similarity to Vif derived from the $\mathrm{C} 1$ isolate that had the lowest infectivity among viruses belonging to subtypes $\mathrm{A}, \mathrm{B}, \mathrm{D}, \mathrm{F}$ and $G$ that were studied [10]. The impact of Vif sequence variations on the Vif-A3G axis remains to be determined. Another possible reason for differences in Vif anti-A3G activity reported by us and others may be the experimental system used to evaluate this activity. Here, we employed a physiological system in which the viral backbone is full-length and subtype-specific, whereas others employed a read-out system that may be less rigorous than the assay described here or have produced the recombinant virions on a subtype B backbone [10, 11]. Additionally, all our recombinant clones retained their subtype-specific envelopes and displayed R5 tropism, in contrast to the pseudotyped virus approach used by others [11]. Furthermore, the target cells used in this study (TZM-bl) express both co-receptors required

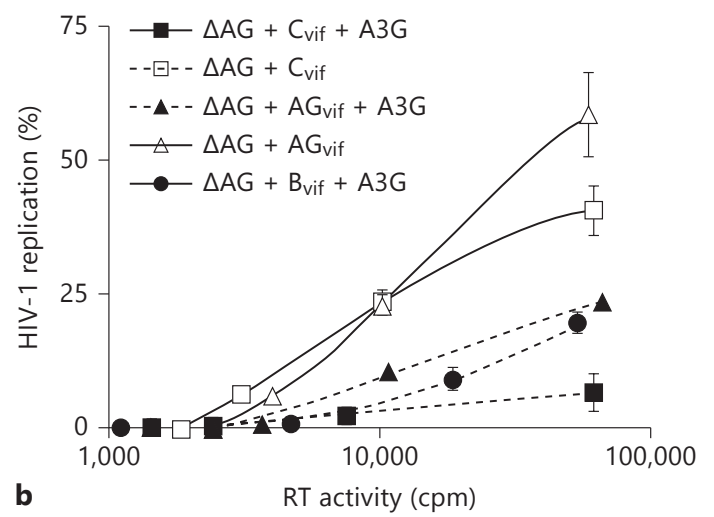

represent replication of viruses produced in the absence of A3G. The dashed lines represent replication of viruses produced in the presence of A3G. Data depict the results of 3 independent experiments performed in duplicate \pm SD.

for HIV-1 entry. This experimental design permits us to study how Vif can overcome A3G-mediated restriction in the course of HIV-1 replication.

Analysis of Vif structural and functional domains revealed that the greatest degree of variation was present in the Vif nuclear localization inhibitory sequence and phosphorylation sites (table 1) [14, 15]. Phosphorylation is known to play an important role in Vif function and regulation of HIV-1 replication [16]. Phosphorylation of Vif serine-144, part of the SLQYLA domain, inhibits elongin $C$ binding and this interferes with A3G ubiquitination [4]. However, the impact of Vif phosphorylation on HIV-1 replication or infectivity at other phosphorylation sites has yet to be determined. We also observed additional variability at specific locations outside the previously defined conserved domains (table 1). A number of these variations were present in Vif sequences derived from the molecular clones that we employed (fig. 2). Further evaluation of such sequence variations will be necessary to mechanistically understand the basis for Vif subtype variability in the context of A3G restriction.

HIV-1 heterogeneity is due in part to lack of RT proofreading ability and the high rate of $\mathrm{HIV}-1$ replication and recombination. The ability of $\mathrm{A} 3 \mathrm{G}$ to introduce mutations into viral DNA may also contribute to viral diversity and impact viral pathogenesis. High-level inhibition of A3G may reduce the HIV mutation rate and generate a more genetically stable virus population, which may 
Table 1. Analysis of Vif functional and structural domains among different HIV-1 subtypes and CRFs

\begin{tabular}{|c|c|c|c|c|c|c|c|}
\hline Subtype & $\begin{array}{l}5,11,21 \\
38 \mathrm{~W}^{\mathrm{a}}\end{array}$ & $\begin{array}{l}\mathrm{HH} \\
42-43^{\mathrm{b}}\end{array}$ & $90-93^{c}$ & $\begin{array}{l}\text { S95, T96, S165, T170 } \\
\text { and T188 }\end{array}$ & $\begin{array}{l}\mathrm{H} 108, \mathrm{C} 114, \\
\mathrm{C} 133 \text { and } \mathrm{H} 139^{\mathrm{e}}\end{array}$ & $144-149^{f}$ & $161-164^{\mathrm{g}}$ \\
\hline $\mathrm{A}_{(\mathrm{n}=80)}{ }^{1}$ & $-{ }^{2}$ & - & 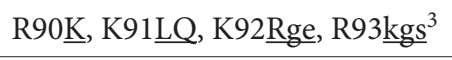 & 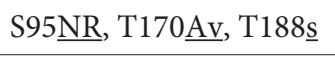 & - & - & - \\
\hline $\mathrm{B}_{(\mathrm{n}=410)}$ & $\begin{array}{l}\mathrm{W} 21 \underline{\mathrm{mt}} \\
\mathrm{W} 38 \mathrm{r}\end{array}$ & $\mathrm{H} 42 \mathrm{pq}$ & 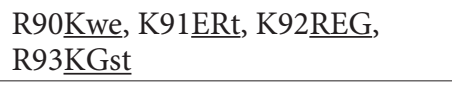 & $\begin{array}{l}\text { S95NRT, S165 } \underline{\mathrm{sn}}, \\
\text { T170무, T188 } \underline{\text { Sip }}\end{array}$ & C114wr & S144다 & $\mathrm{L} 163 \underline{\mathrm{Fv}}$ \\
\hline $\mathrm{C}_{(\mathrm{n}=364)}$ & $\mathrm{W} 11 \underline{\mathrm{r}}$ & $\mathrm{H} 42 \underline{\mathrm{n}}$ & 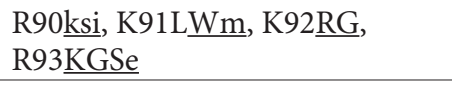 & S95스, T170스, T188I & - & - & - \\
\hline $\mathrm{D}_{(\mathrm{n}=49)}$ & - & - & 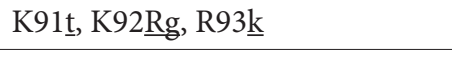 & S95므 & - & - & - \\
\hline $\mathrm{F}_{(\mathrm{n}=16)}$ & - & - & K91ㅁ, K92ㅁ, R93nㅡ & S95ㅁ, T170믄 & - & - & - \\
\hline $\mathrm{G}_{(\mathrm{n}=7)}$ & - & $\mathrm{H} 42 \mathrm{p}$ & 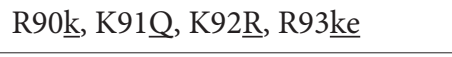 & T170 & - & - & - \\
\hline $\mathrm{H} \mathrm{J} \mathrm{K}_{(\mathrm{n}=7)}$ & - & - & K91lq, K92er, R93k & S95ㄹk, T170트 & - & S144hㅡ & $\mathrm{L} 163 \underline{\mathrm{Vk}}$ \\
\hline
\end{tabular}

a Tryptophan-rich stretch - involved in A3G and A3F binding. ${ }^{\text {b }}$ Hydrophobic motif involved in A3G interaction (also requires amino acids $14-17$ for interacting with A3G). ${ }^{\mathrm{c}}$ NLIS: nuclear localization inhibitory signal, RKKR. ${ }^{\mathrm{d}}$ Phosphorylation sites. ${ }^{\mathrm{e}}$ Zinc-binding motif HCCH. ${ }^{f}$ Viral BC-Box, SLQYLA motif, elonginC binding. ${ }^{\mathrm{g}}$ Vif dimerization domain, PPLP.

${ }^{1} \mathrm{n}$ : number of sequences analyzed. ${ }^{2}(-)$ : no change in sequence was observed. ${ }^{3}$ Lightface type indicates original amino acid at the designated location; underlined type indicates sequence variation from lightface type; Variations found in $\geq 5$ sequences are in capital, and variations found in $\leq 5$ are in lower case.

render it more susceptible to elimination by the immune system $[17,18]$. On the other hand, A3G may increase the mutation rate in HIV, such that error catastrophe occurs from which the virus cannot recover. Subtype variations within Vif may play a role in this dynamic.

In recent years, several small molecules that can help A3G to evade Vif have been described [19, 20]. These molecules antagonize Vif by targeting it for proteasomal degradation and increase $\mathrm{A} 3 \mathrm{G}$ incorporation into the virion, thus increasing the HIV mutation rate. Our findings suggest that Vif variability may have an impact on the Vif-A3G axis. The development of Vif antagonists should be approached with caution in order to maintain the HIV mutation rate in a range that will not provide a replicative benefit to the virus, which may in turn lead to increased HIV-1 pathogenesis.

\section{Acknowledgements}

pAG_97 (AB052867) and pINDIE_C clones (AB023804) were kindly provided by M. Takahoko and N. Mochizuki, respectively. The pNL/ADA clone (AF004394) and pcDNA3.1-APOBEC3G plasmid (BC024268) were obtained from the AIDS Research and Reference Reagent Program, NIAID, NIH (\#11346, \#10102) (Bethesda, Md., USA), with courtesy of Dr. Eric O. Freed and Drs. B. Matija Peterlin and Yong-Hui Zheng, respectively.

We would like to thank Estrella Moyal for her expertise and collegial support. This work was supported by grants from the Canadian Institutes of Health Research. S.M.S. and D.C. were supported by CIHR doctoral fellowship awards. R.D.S. was supported by a postdoctoral fellowship from the CIHR Canadian HIV Trials Network (CTN).

\section{Disclosure Statement}

The authors have no conflicts of interest to disclose.

References
1 Sheehy AM, Gaddis NC, Choi JD, Malim MH: Isolation of a human gene that inhibits HIV-1 infection and is suppressed by the viral Vif protein. Nature 2002;418:646-650.

2 Zhang $\mathrm{H}$, Yang B, Pomerantz RJ, Zhang C, Arunachalam SC, Gao L: The cytidine deaminase CEM15 induces hypermutation in newly synthesized HIV-1 DNA. Nature 2003;424: 94-98.

-3 Mehle A, Strack B, Ancuta P, Zhang C, McPike $M$, Gabuzda D: Vif overcomes the innate antiviral activity of APOBEC3G by promoting its degradation in the ubiquitin-proteasome pathway. J Biol Chem 2004;279:7792-7798.

4 Malim MH, Bieniasz PD: HIV restriction factors and mechanisms of evasion. Cold Spring Harb Perspect Med 2012;2:a006940. 
5 Wei M, Xing H, Hong K, Huang H, Tang H, Qin G, Shao Y: Biased G-to-A hypermutation in HIV-1 proviral DNA from a long-term non-progressor. AIDS 2004;18:1863-1865.

-6 Zhang L, Huang Y, Yuan H, Tuttleton S, Ho DD: Genetic characterization of vif, vpr, and vpu sequences from long-term survivors of human immunodeficiency virus type 1 infection. Virology 1997;228:340-349.

-7 Tian C, Yu X, Zhang W, Wang T, Xu R, Yu XF: Differential requirement for conserved tryptophans in human immunodeficiency virus type $1 \mathrm{Vif}$ for the selective suppression of APOBEC3G and APOBEC3F. J Virol 2006; 80:3112-3115.

8 Korber B, Gaschen B, Yusim K, Thakallapally R, Kesmir C, Detours V: Evolutionary and immunological implications of contemporary HIV-1 variation. Br Med Bull 2001;58: $19-42$.

9 Taylor BS, Sobieszczyk ME, McCutchan FE, Hammer SM: The challenge of HIV-1 subtype diversity. N Engl J Med 2008;358:1590-1602.

10 Binka M, Ooms M, Steward M, Simon V: The activity spectrum of Vif from multiple HIV-1 subtypes against APOBEC3G, APOBEC3F, and APOBEC3H. J Virol 2012;86:49-59.
11 Iwabu Y, Kinomoto M, Tatsumi M, Fujita H, Shimura M, Tanaka Y, Ishizaka Y, Nolan D, Mallal S, Sata T, Tokunaga K: Differential anti-APOBEC3G activity of HIV-1 Vif proteins derived from different subtypes. J Biol Chem 2010;285:35350-35358.

12 Takahoko M, Tobiume M, Ishikawa K, Ampofo W, Yamamoto N, Matsuda M, Tatsumi M: Infectious DNA clone of HIV type $1 \mathrm{~A} / \mathrm{G}$ recombinant (CRF02_AG) replicable in peripheral blood mononuclear cells. AIDS Res Hum Retroviruses 2001;17:1083-1087.

13 Lisovsky I, Schader SM, Martinez-Cajas JL, Oliveira M, Moisi D, Wainberg MA: HIV-1 protease codon 36 polymorphisms and differential development of resistance to nelfinavir, lopinavir, and atazanavir in different HIV-1 subtypes. Antimicrob Agents Chemother 2010;54:2878-2885.

14 He Z, Zhang W, Chen G, Xu R, Yu XF: Characterization of conserved motifs in HIV-1 Vif required for $\mathrm{APOBEC} 3 \mathrm{G}$ and $\mathrm{APOBEC} 3 \mathrm{~F}$ interaction. J Mol Biol 2008;381:1000-1011.
5 Zhang W, Chen G, Niewiadomska AM, Xu R, Yu XF: Distinct determinants in HIV-1 Vif and human APOBEC3 proteins are required for the suppression of diverse host anti-viral proteins. PLoS One 2008;3:e3963.

16 Mehle A, Goncalves J, Santa-Marta M, McPike M, Gabuzda D: Phosphorylation of a novel SOCS-box regulates assembly of the HIV-1 Vif-Cul5 complex that promotes APOBEC3G degradation. Genes Dev 2004;18:2861-2866.

17 Harris RS: Enhancing immunity to HIV through APOBEC. Nat Biotechnol 2008;26:10891090.

18 Pillai SK, Wong JK, Barbour JD: Turning up the volume on mutational pressure: is more of a good thing always better? A case study of HIV1 Vif and APOBEC3. Retrovirology 2008;5:26.

19 Cen S, Peng Z, Li X, Li Z, Ma J, Wang Y, Fan B, You X, Wang Y, Liu F, Shao R, Zhao L, Yu L, Jiang J: Small molecular compounds inhibit HIV-1 replication through specifically stabilizing APOBEC3G. J Biol Chem 2010;285: 16546-16552.

20 Nathans R, Cao H, Sharova N, Ali A, Sharkey M, Stranska R, Stevenson M, Rana TM: Smallmolecule inhibition of HIV-1 Vif. Nat Biotechnol 2008;26:1187-1192. 